\title{
Risk and protective factors for the occurrence of sporadic pancreatic endocrine neoplasms
}

\author{
Roberto Valente', Alastair J Hayes², Sven-Petter Haugvik3, Per Hedenström4, \\ Darko Siuka5, Emilie Korsæth3, Daniel Kämmerer6, Stuart M Robinson7, \\ Patrick Maisonneuve ${ }^{8}$, Gianfranco Delle Fave', Bjorn Lindkvist4 and

\section{Gabriele Capurso'}

1Digestive and Liver Disease Unit, Sant' Andrea Hospital, Sapienza University of Rome, Rome, Italy 2Department of General Surgery, Royal Infirmary of Edinburgh, Edinburgh, UK 3Department of Hepato-Pancreato-Biliary Surgery, Oslo University Hospital, Oslo, Norway

${ }^{4}$ Unit of Gastroenterology, Department of Medicine, Sahlgrenska University Hospital, Gothenburg, Sweden ${ }^{5}$ Department of Gastroenterology, University Medical Centre Ljubljana, Ljubljana, Slovenia ${ }^{6}$ Department of General and Visceral Surgery, Zentralklinik Bad Berka, Bad Berka, Germany ${ }^{7}$ Department of Hepatopancreatobiliary and Transplantation Surgery, The Freeman Hospital, Newcastle upon Tyne, UK

${ }^{8}$ Division of Epidemiology and Biostatistics, European Institute of Oncology, Milan, Italy

Correspondence should be addressed to $\mathrm{G}$ Capurso Email gabriele.capurso@gmail.com

\begin{abstract}
Pancreatic neuroendocrine neoplasms (PNENs) represent $10 \%$ of all pancreatic tumors by prevalence. Their incidence has reportedly increased over recent decades in parallel with that of pancreatic adenocarcinoma. PNENs are relatively rare, and of the few institutions that have published potential risk factors, findings have been heterogeneous. Our objective was to investigate the association between potential risk and protective factors for the occurrence of sporadic PNENs across a European population from several institutions. A multinational European case-control study was conducted to examine the association of selected environmental, family and medical exposure factors using a standardized questionnaire in face-to-face interviews. A ratio of $1: 3$ cases to controls were sex and age matched at each study site. Adjusted univariate and multivariate logistic regression analysis were performed for statistically significant factors. The following results were obtained: In 201 cases and 603 controls, non-recent onset diabetes (OR 2.09, $\mathrm{Cl}$ 1.27-3.46) was associated with an increased occurrence of PNENs. The prevalence of non-recent onset diabetes was higher both in cases with metastatic disease (TNM stage III-IV) or advanced grade (G3) at the time of diagnosis. The use of metformin in combination with insulin was also associated with a more aggressive phenotype. Drinking coffee was more frequent in cases with localized disease at diagnosis. Our study concluded that non-recent onset diabetes was associated with an increased occurrence of PNENs and the combination of metformin and insulin was consistent with a more aggressive PNEN phenotype. In contrast to previous studies, smoking, alcohol and first-degree family history of cancer were not associated with PNEN occurrence.
\end{abstract}

\section{Key Words}

- pancreas

- neuroendocrine neoplasms

- insulinomas

- gastrinomas

- risk factors 


\section{Introduction}

Pancreatic neuroendocrine neoplasms (PNENs) are a group of tumors which originate from endocrine cells within the pancreas gland. PNENs have heterogeneous clinical behavior owing to their hormone functional status, cellular characteristics and the extent of metastatic disease. While representing only 1-2\% of pancreatic neoplasms by incidence (Fitzgerald et al. 2008, Yao et al. 2008), PNENs may account for as much as $10 \%$ by prevalence (Yao et al. 2008). Such discrepancy is due in part to the relatively indolent clinical course of many PNENs compared to pancreatic adenocarcinoma. PNENs are rare tumors but their incidence has reportedly increased in recent decades, particularly that of non-functioning tumors (Lepage et al. 2004, Fitzgerald et al. 2008, Yao et al. 2008). To a lesser degree, investigators have also identified a modest increase in the incidence of pancreatic adenocarcinoma (Fitzgerald et al. 2008).

The increased reported incidence of both endocrine and exocrine pancreatic tumors is likely due to rising population lifespan and the wider availability of highresolution cross-sectional imaging (Del Chiaro et al. 2013, Ellison et al. 2014), but additionally raises the possibility of changing exposure to factors which may alter the risk of pancreatic neoplasia.

There are a small number of case-control studies that have investigated potential risk factors for the occurrence of PNENs (Hassan et al. 2008b, Capurso et al. 2009, Zhan et al. 2013, Halfdanarson et al. 2014, Ben et al. 2016). These studies recruited participants from a single institution or geographical region, and were recently summarized in a meta-analysis that found personal history of diabetes mellitus and family history of cancer to be associated with an increased risk of PNEN (Haugvik et al. 2015). The association of PNENs with smoking and alcohol drinking was less clear, and only heavy smoking and heavy alcohol consumption reached statistical significance. The included studies differed in their design and population definitions, with a considerable heterogeneity limiting the significance of the meta-analysis. Moreover, these studies had some specific methodological limitations, such as the absence of a power calculation and exposures were often recorded at the time of diagnosis or treatment, rather than considering the exposure history prior to diagnosis. The latter raises the possibility of a bias due to cancer symptoms (e.g. weight loss, new onset diabetes) or lifestyle modifications such as changes in smoking behavior and alcohol consumption.
It appears that there is overlap in risk factors, such as smoking and alcohol, for the occurrence of PNENs and pancreatic adenocarcinoma; however, a number of other factors that have been associated with the occurrence of pancreatic cancer have not, to our knowledge, been investigated for PNENs. For example, the use of medications such as aspirin, and the association with allergy and atopy that have been reported to be protective against pancreatic adenocarcinoma occurrence (Streicher et al. 2014, Gomez-Rubio et al. 2017).

For these reasons, we conducted a multicenter European study aimed at assessing the association between a large number of potential risk or protective factors for the development of sporadic PNENs.

\section{Materials and methods}

\section{Study design and population}

A collaborative multicenter hospital-based case-control study was conducted in six European countries: Italy, Norway, Sweden, Slovenia, United Kingdom and Germany as part of the 'Pancreas 2000' educational project (www.pancreas2000.org), upon local hospital ethical committee approval.

A standardized questionnaire, including questions about demographics and potential risk factors, such as family history of cancer, environmental factors, previous use of drugs and other medical history features was administered to patients by a trained medical doctor. Each questionnaire took 15 min to be completed during a face-to-face interview, after gaining participant consent.

The cases were prevalent sporadic PNEN patients diagnosed within 24 months from the beginning of the study (January 2013) and new incident cases of sporadic PNENs diagnosed from January 2013 to December 2015 that were recruited at the participating centers.

The inclusion criterion was to have a histological or cytological diagnosis of PNEN. The date of the confirmatory pathological report was accepted as the date of diagnosis.

Exclusion criteria were the presence of an inherited form of PNEN such as those associated with multiple endocrine neoplasia-type 1 (MEN1), von Hipple-Lindau disease (VHL), neurofibromatosis type 1 (NF1), tuberous sclerosis (TSC) or an inability to participate, such as dementia.

Published by Bioscientifica Ltd. 
According to the absence or presence of a clinical syndrome due to hormonal hypersecretion, cases were classified as non-functioning or functioning tumors (gastrinoma, insulinoma, glucagonoma). For example, the Zollinger Ellison Syndrome was clinically suspected in the presence of a PNEN associated with peptic disease and its complications and diarrhea, insulinomas in the presence of severe hypoglycemia with associated neurological symptoms (varying from confusion to coma), glucagonoma in the presence of rash, glucose intolerance and weight loss. In any case, the syndrome was confirmed with a specific laboratory workup according to guidelines (Jensen et al. 2012, Falconi et al. 2016).

Cases were classified according to the European Neuroendocrine Tumor Society (ENETs) and the 2010 World Health Organization classifications (Rindi et al. 2010, Solcia et al. 2002, Falconi et al. 2016).

Eligible controls were either individuals seen in the participating hospitals' outpatient clinic for a non-specific, non-organic gastrointestinal disorder (bloating, aspecific dyspeptic symptoms, eructation) or visitors attending the same network of referring hospitals, matched by country, sex and age (+/- 5 years). Visitors and hospital outpatients' clinic belonged to the same catchment area of cases. Specific exclusion criteria for controls were the following: (1) the presence of any genetic syndrome associated with the occurrence of PNENs; (2) a history of active cancer (diagnosed within 5 years); (3) any biological relation of a participating PNEN case in this study; (4) a history of any chronic inflammatory condition (e.g. chronic obstructive pulmonary disease, liver cirrhosis, inflammatory bowel disease, end stage kidney disease); and (5) undergoing evaluation of a possible familial cancer syndrome. Controls were included in the same country and interviewed within 6 months of the inclusion of the matched corresponding case.

\section{Exposure definitions}

Subjects were questioned about risk factors that were present at least 12 months before diagnosis or presentation of symptoms, in order to avoid potential bias due to lifestyle modifications, cancer symptoms or cancer treatments.

Subjects were considered ever smokers if they reported a cumulative lifetime smoking history greater than 6 months or 100 cigarettes smoked. A quantification of the smoking habit for cases and controls was performed considering the number of pack-years (pack-year = number of packs per day $\times$ years of smoking), with 20 packyears being the lower limit to qualify a participant a heavy smoker.

A daily intake of at least $12.5 \mathrm{~g}$ of alcohol, equivalent to one glass of wine, one pint of beer or one shot of hard liquor, for at least one year, was considered the cut-off to be a regular ever alcohol drinker. Because of possible different drinking habits within different European countries, the weekly alcohol amount was also sub-analyzed according to low (1-7 weekly units assumption), medium (8-14 weekly units), medium-high (14-20 weekly units) and heavy alcohol consumption ( $\geq 21$ weekly units). Coffee drinking was also recorded as ever drinking (at least one cup per day) or heavy coffee drinking ( $>5$ cups per day).

Height and weight were recorded from which body mass index (BMI) $\left(\mathrm{kg} / \mathrm{m}^{2}\right)$ was calculated. A history of chronic pancreatitis, acute pancreatitis, peptic ulcer disease, biliary stones and previous surgery were specifically recorded. Additionally, a diagnosis of diabetes was documented and subdivided for type and onset. For cases, recent onset diabetes was defined as that which was diagnosed in 12 months prior to the PNEN diagnosis, and for controls, a diagnosis of diabetes 12 months prior to the date of recruitment was required. Sensitivity analysis was also performed for different intervals of the onset of diabetes, (inferior to 1 year, between 1 and 3 years, between 3 and 5 years and above 5 years, respectively). Another sensitivity analysis was also conducted considering incident/prevalent cases and hospitals controls/visitors controls compared to respective cases.

As atopy and allergy have been associated with a reduced risk of pancreatic cancer (Gomez-Rubio et al. 2017), cases and controls were interrogated about a history of allergy, with specific enquiry for eczema, hay fever and asthma. The use of aspirin, proton pump inhibitors, metformin and insulin were recorded. Subjects were interrogated about 1st and 2nd degree family history of cancer, and the total number of siblings and children was recorded.

\section{Statistical analysis}

An a priori power calculation was performed. We estimated sample size based on the differences reported in the frequencies of exposure in cases and controls according to a previous study (Capurso et al. 2009), and considering a ratio $1: 3$, with a statistical power equal to $80 \%$ and an alpha error equal to 0.05 . 
According to Capurso and coworkers (Capurso et al. 2009) the reported prevalence in cases and controls was, respectively, $53 \%$ vs $32 \%$ for 1 st degree family history of cancer, $10 \%$ vs $2 \%$ for diabetes and $14 \%$ vs $3 \%$ for heavy alcohol consumption. Therefore, the sample size's estimate of cases and controls, to show true differences whether existing, were, respectively, 64 cases and 191 controls for 1st degree family history of cancer, 117 cases and 350 controls for diabetes, and 85 cases and 253 controls for heavy alcohol consumption.

We therefore estimated that a total of 200 cases and 600 controls across participating centers to be an adequate sample size to reveal differences in the prevalence of most risk factors analyzed among cases and controls, where these exist.

Characteristics of cases and controls were compared by chi-square test for categorical variables or Student's $t$-test for continuous variables. Significant variables were analyzed by logistic regression analysis adjusted for sex, age and enrollment center. A multivariable logistic regression analysis, adjusted for sex, age and enrollment center was also performed with an enter selection procedure for statistically significant risk factors. A dedicated statistical software package (MedCalc, Mariakerke, Belgium) was used for data analysis. Ninety-five percent confidence interval (95\%) was calculated where possible. All $P$ values were two sided, and a $P<0.05$ was considered statistically significant.

\section{Results}

\section{Characteristics of cases and controls}

A total of 201 cases and 603 sex- and age-matched controls were enrolled among the six centers (Italy, Norway, Sweden, United Kingdom, Slovenia and Germany) as shown in Table 1. The mean age was 59.6 years in cases (CI 57.7-61.4) and 59.5 years in controls (CI 58.4-60.55), and $51 \%$ of cases and controls were male.

Among the 201 PNEN cases, 154 (76.6\%) had a nonfunctioning tumor. Of the 47 functioning PNENs, 26 (55.3\%) had an insulinoma, 9 (19.1\%) a gastrinoma, 7 (14.9\%) a glucagonoma and 5 (10.6\%) other functioning tumors. The majority were G1 $(44.8 \%)$ or G2 (43.3\%) PNENs, and were equally distributed among different disease stages (Table 1). Of the 201 PNEN cases, 80 (38\%) cases were incident and 121 (62\%) prevalent. Of the 603 controls, $422(70 \%)$ were hospital outpatients controls and $181(30 \%)$ visitors.
Table 1 Characteristics of PNENs cases.

\begin{tabular}{|c|c|}
\hline & Cases $\boldsymbol{N}(\%)$ \\
\hline Total & 201 \\
\hline \multicolumn{2}{|l|}{ Referral center } \\
\hline Italy & $62(30.8)$ \\
\hline Norway & $44(21.9)$ \\
\hline Sweden & $40(19.9)$ \\
\hline Slovenia & $24(11.9)$ \\
\hline United Kingdom & $20(10.0)$ \\
\hline Germany & $11(5.5)$ \\
\hline \multicolumn{2}{|l|}{ Sex } \\
\hline Male & $103(51.2)$ \\
\hline \multicolumn{2}{|l|}{ Age (years) } \\
\hline Mean \pm S.D. & $59.6 \pm 13.1$ \\
\hline Race Caucasians & $196(97.5)$ \\
\hline \multicolumn{2}{|l|}{ Tumor type } \\
\hline Functioning & $47(23.3)$ \\
\hline Non-functioning & $154(76.7)$ \\
\hline \multicolumn{2}{|c|}{ Functioning only $(n=47)$} \\
\hline Insulinomas & $26(55.3)$ \\
\hline Gastrinomas & $9(19.1)$ \\
\hline Glucagonomas & $7(14.9)$ \\
\hline Other & $5(10.6)$ \\
\hline \multicolumn{2}{|l|}{ Tumor grade } \\
\hline G1 & $90(44.8)$ \\
\hline $\mathrm{G} 2$ & $87(43.3)$ \\
\hline G3 & $22(10.9)$ \\
\hline Unknown & $2(1.0)$ \\
\hline \multicolumn{2}{|l|}{ Tumor stage } \\
\hline Stage I & $51(25.4)$ \\
\hline Stage II & $53(26.4)$ \\
\hline Stage III & $47(23.4)$ \\
\hline Stage IV & $43(21.4)$ \\
\hline Unknown & $7(3.5)$ \\
\hline \multicolumn{2}{|l|}{ Tumor site } \\
\hline Head & $75(37.3)$ \\
\hline Body or tail & $120(59.7)$ \\
\hline Unknown & $6(3.0)$ \\
\hline
\end{tabular}

\section{Risk factors for the occurrence of PNEN}

Family history of cancer The proportion of subjects who had a 1st degree family history of cancer was similar both in cases and in controls $(51.1 \%$ vs $45.3 \% P=0.17$, respectively), while 2 nd degree family history was slightly more prevalent in cases $(36.8 \%$ vs $30.2 \% P=0.09)$. A 1 st degree family history of specific cancer sites was also not significantly different (Table 2). No cases or controls reported a family history of neuroendocrine tumor (NET). At multiple regression analysis, $2^{\text {nd }}$ degree family history of any cancer was, however, associated with an increased risk of PNEN (Tables 2 and 3).

Body mass index Mean BMI was not significantly different among cases and controls $\left(26.8 \mathrm{~kg} / \mathrm{m}^{2}\right.$ (CI 26.0 27.5 ) and $26.4 \mathrm{~kg} / \mathrm{m}^{2}$ (CI 26.4-26.8), $P=0.10$. Similarly, while the overall prevalence of obesity was more frequent

Published by Bioscientifica Ltd 
Table 2 Risk factors for PNENs.

\begin{tabular}{|c|c|c|c|c|}
\hline & Cases $\boldsymbol{N}(\%)$ & Controls $\boldsymbol{N}(\%)$ & $P$ value & OR* $(95 \% \mathrm{Cl})$ \\
\hline Total & 201 & 603 & & \\
\hline \multicolumn{5}{|l|}{ Family history* } \\
\hline 1st degree FH of any cancer & $103(51.1)$ & $272(45.3)$ & 0.17 & $1.32(0.94-1.83)$ \\
\hline 2nd degree $\mathrm{FH}$ of any cancer & $74(36.8)$ & $181(30.2)$ & 0.09 & $1.51(1.05-2.17)$ \\
\hline 1st degree FH pancreatic cancer & $6(3.0)$ & $13(2.2)$ & 0.69 & $1.42(0.52-3.84)$ \\
\hline 1st degree FH esophageal & $2(1.0)$ & $4(0.7)$ & 0.99 & $1.58(0.28-8.79)$ \\
\hline 1st degree FH gastric cancer & $7(3.5)$ & $26(4.3)$ & 0.75 & $0.77(0.32-1.85)$ \\
\hline 1st degree FH colorectal cancer & $18(9.0)$ & $57(9.5)$ & 0.92 & $0.99(0.56-1.75)$ \\
\hline 1st degree FH breast cancer & $20(10.0)$ & $51(8.3)$ & 0.62 & $1.25(0.72-2.18)$ \\
\hline 1st degree FH lung cancer & $17(8.5)$ & $48(8.0)$ & 0.95 & $1.12(0.62-2.00)$ \\
\hline 1st degree FH NETs & - & - & & \\
\hline 1st degree FH hematological cancer & $12(6.0)$ & $30(5.0)$ & 0.72 & $1.28(0.63-2.57)$ \\
\hline 1st degree FH hepatobiliary cancer & $4(2.0)$ & $17(2.8)$ & 0.69 & $0.45(0.12-1.66)$ \\
\hline 1st degree FH sarcoma & - & $3(0.5)$ & 0.73 & - \\
\hline \multicolumn{5}{|l|}{ Number of siblings } \\
\hline Mean \pm S.D. & $2.1 \pm 1.7$ & $2.5 \pm 2.0$ & 0.05 & \\
\hline \multicolumn{5}{|l|}{ BMI } \\
\hline Underweight & $5(2.5)$ & $10(1.6)$ & & $1.68(0.55-5.04)$ \\
\hline Normal weight & $82(41.0)$ & $243(40.2)$ & P-trend ${ }^{* *}$ & $1.00(0.71-1.39)$ \\
\hline Overweight & $63(31.5)$ & $240(39.8)$ & 0.44 & $0.75(0.52-1.10)$ \\
\hline Obese & $51(25.5)$ & $110(18.2)$ & & $1.36(0.88-2.08)$ \\
\hline \multicolumn{5}{|l|}{ Smoking } \\
\hline Never smoke & $89(44.5)$ & $279(46.5)$ & & $0.86(0.62-1.20)$ \\
\hline$<20$ pack-years & $60(30.0)$ & $176(29.3)$ & P-trend & $1.07(0.75-1.53)$ \\
\hline$\geq 20$ pack-years & $51(25.5)$ & $144(24.0)$ & 0.59 & $1.15(0.78-1.69)$ \\
\hline Unknown & $1(0.5)$ & $3(0.5)$ & & 1.00 \\
\hline \multicolumn{5}{|l|}{ Alcohol intake } \\
\hline Never drink & $49(24.4)$ & $173(29.2)$ & & $0.77(0.53-1.12)$ \\
\hline$<21$ units/week & $145(72.1)$ & $394(66.6)$ & P-trend ${ }^{* *}$ & $1.29(0.90-1.85)$ \\
\hline$\geq 21$ units/week & $7(3.4)$ & $25(4.2)$ & 0.33 & $0.88(0.37-2.11)$ \\
\hline \multicolumn{5}{|l|}{ Coffee } \\
\hline Never drink & $30(15.2)$ & 73 (12.2) & & $1.37(0.83-2.14)$ \\
\hline$\leq 4$ cups/day & $135(68.2)$ & $411(68.7)$ & P-trend** & $0.94(0.66-1.34)$ \\
\hline$>4$ cups/day & $33(18.8)$ & $114(19.2)$ & 0.24 & $0.86(0.55-1.35)$ \\
\hline Unknown & $3(1.4)$ & $5(0.8)$ & & 1.00 \\
\hline \multicolumn{5}{|l|}{ Diabetes } \\
\hline No & $164(81.6)$ & $529(87.7)$ & 0.03 & 1.00 \\
\hline Recent onset ( $<1$ year) & $1(0.5)$ & $10(1.6)$ & 0.4 & $0.24(0.03-1.88)$ \\
\hline No-recent onset ( $\geq 1$ year) & $35(17.4)$ & $58(9.7)$ & 0.004 & $1.89(1.17-3.05)$ \\
\hline Unknown & $1(0.5)$ & $6(1.0)$ & & 1.00 \\
\hline Diabetes ( $\geq 1$ year, $<3$ years) & $6(3.0)$ & $7(1.1)$ & 0.07 & $2.56(0.83-7.91)$ \\
\hline Diabetes ( $\geq 3$ year, $<5$ years) & $8(4.0)$ & $6(0.9)$ & 0.0045 & $4.31(1.43-12.98)$ \\
\hline Diabetes ( $\geq 5$ years) & $21(10.7)$ & $45(7.4)$ & 0.16 & $1.47(0.84-2.56)$ \\
\hline \multicolumn{5}{|l|}{ Diabetes treatment } \\
\hline Metformin (no insulin) & $13(7.3)$ & $29(5.1)$ & 0.3 & $1.35(0.68-2.66)$ \\
\hline Insulin & $7(4.1)$ & 9 (1.6) & 0.1 & $1.63(0.86-3.08)$ \\
\hline Metformin and insulin & $9(5.2)$ & $19(3.4)$ & 0.4 & $1.48(0.65-3.35)$ \\
\hline \multicolumn{5}{|l|}{ Past medical history } \\
\hline Acute pancreatitis & $7(3.5)$ & $15(2.5)$ & 0.60 & $1.42(0.56-3.60)$ \\
\hline Chronic pancreatitis & - & - & - & \\
\hline Peptic ulcer & $24(12.3)$ & $64(10.6)$ & 0.59 & $1.25(0.75-2.07)$ \\
\hline Cholecystectomy & $18(9.0)$ & $51(8.5)$ & 0.92 & $1.07(0.61-1.90)$ \\
\hline Gastrectomy & $2(1.0)$ & $4(0.7)$ & 0.99 & $1.56(0.28-8.65)$ \\
\hline Gallstone disease & $34(19.2)$ & $77(13.3)$ & 0.06 & $1.53(0.97-2.42)$ \\
\hline Asthma & $24(12.1)$ & $52(8.6)$ & 0.19 & $1.47(0.87-2.47)$ \\
\hline Eczema & $22(11.1)$ & $46(7.6)$ & 0.17 & $1.52(0.88-2.62)$ \\
\hline Hay fever & $31(15.6)$ & $87(14.5)$ & 0.79 & $1.17(0.74-1.84)$ \\
\hline Any allergy & $58(28.9)$ & $151(25.0)$ & 0.32 & $1.26(0.88-1.82)$ \\
\hline Use of aspirin & $45(22.5)$ & $160(26.5)$ & 0.29 & $0.79(0.53-1.18)$ \\
\hline Use of proton pump inhibitors & $78(39.2)$ & $239(39.6)$ & 0.97 & $1.04(0.74-1.45)$ \\
\hline
\end{tabular}

*OR adjusted for sex, age and center of enrollment; ${ }^{*} P$ value based on the Mantel-Haenszel test for trend excluding missing category. $\mathrm{FH}$, family history; NET, neuroendocrine tumor.

http://erc.endocrinology-journals.org DOI: 10.1530/ERC-17-0040
() 2017 Society for Endocrinology Printed in Great Britain 
Table 3 Risk factors for the occurrence of PNENs at the Logistic regression analysis.

\begin{tabular}{|c|c|c|c|c|}
\hline Risk factor & Univariate* OR $(95 \% \mathrm{Cl})$ & $P$ value & Multivariable* OR $(95 \% \mathrm{Cl})$ & $P$ value \\
\hline \multicolumn{5}{|l|}{ Diabetes } \\
\hline No & 1.00 & & 1.00 & \\
\hline Early onset ( $\leq 1$ year) & $0.24(0.03-1.88)$ & 0.17 & & \\
\hline Late onset (>1 year) & $1.89(1.17-3.05)$ & 0.008 & $2.09(1.27-3.45)$ & 0.003 \\
\hline \multicolumn{4}{|c|}{ 2nd degree FH of any cancer } & 0.03 \\
\hline \multicolumn{5}{|l|}{ Gallstone disease } \\
\hline No & 1.00 & & & \\
\hline Yes & $1.48(0.93-2.35)$ & 0.08 & $1.52(0.95-2.44)$ & 0.08 \\
\hline
\end{tabular}

Adjusted for sex, age, underweight, 1st degree family history for esophageal cancer and center of enrollment.

in cases than controls $(25.5 \%$ vs $18.2 \%)$, this was not significant $(P=0.44)$.

At regression analysis after adjustment for matching variables, there remained no significant association with obesity (OR 1.36, 95\% CI 0.88-2.08, $P=0.15$ ) (Table 3).

\section{Cigarette smoking, alcohol intake and coffee} drinking The proportion of smokers (55.5\% vs $53.5 \%$, $P=0.59)$, heavy smokers $(25.5 \%$ vs $24.0 \%, P=0.59)$, alcohol drinkers $(75.6 \%$ vs $70.8 \%, P=0.33)$, heavy alcohol drinkers (3.4\% vs $4.2 \%, P=0.33)$, coffee drinkers $(84.8 \%$ vs $87.8 \%, P=0.24)$ and heavy coffee drinkers $(18.8 \%$ vs $19.2 \%, P=0.24$ ) did not significantly differ between cases and controls (Table 2 ).

History of diabetes mellitus A history of diabetes mellitus was more prevalent in cases than in controls (18.4\% vs $12.3 \%, P=0.03$ ). This difference was greater on analysis of non-recent onset diabetes, defined as diabetes diagnosed more than 12 months before the diagnosis of PNEN in cases, or 12 months prior to the interview for controls $(17.4 \%$ vs 9.7\%, $P=0.004)$ (Table 2). After adjustment for the matching variables, non-recent onset diabetes was confirmed to be consistent with the occurrence of PNEN (OR 1.89, 95\% CI 1.17-3.05, $P=0.008$ ). At multivariable analysis, the association with non-recent onset diabetes remained statistically significant (OR 2.09, 95\% CI 1.27-3.45, $P=0.003$ ) (Table 3). At sensitivity analysis, a history of diabetes with an onset between 1-3 year and 3-5 years was increasingly prevalent in PNEN compared to controls $(3.0 \%$ vs $1.1 \% P=0.07$ and $4 \%$ vs $0.9 \% P=0.004$, respectively). At univariable logistic regression analysis, this difference remained significant (OR 2.56, 95\% CI 0.83-7.91 and OR 4.31, 95\% CI 1.4312.98 , respectively). For intervals of occurrence of diabetes superior to 5 years, no statistically significant difference was found between cases and controls (10.7\% vs $7.4 \%$,
$P=0.16$, respectively) (Table 2 ). We also performed a separate analysis for 'late onset diabetes' using as controls either only the 422 hospital controls or only the 181 visitors controls. In the first case, the OR resulted to be 2.52 (95\% CI 1.08-5.86; $P=0.03)$, while in the second one, the OR was 1.7 (95\% CI 0.95-3; $P=0.07)$, most likely due to the lower number of controls reducing the power of the analysis.

The use of metformin $(7.3 \%$ vs $5.1 \%, P=0.3)$ and insulin $(4.1 \%$ vs $1.6 \%, P=0.1)$ or their association together (5.2\% vs $3.4 \%, P=0.4)$ was not statistically different between cases and controls.

Past medical history With regard to past medical history, the prevalence of acute pancreatitis $(3.5 \%$ vs $2.4 \%, P=0.60)$, peptic ulcer disease $(12.3 \%$ vs $10.6 \%$, $P=0.59)$, cholecystectomy $(9.0 \%$ vs $8.5 \%, P=0.92)$ and gastrectomy $(1.0 \%$ vs $0.7 \%, P=0.99)$ were similar in cases and controls. None of the participants reported a medical history of chronic pancreatitis (Table 2). A higher proportion of cases reported a history of gallstone disease than controls $(19.2 \%$ vs $13.3 \%)$ but this did not reach the significance threshold $(P=0.06)$. After adjustment for age, sex and enrollment center at multivariable analysis, this latter association remained borderline significant (OR 1.52, 95\% CI 0.95-2.44, $P=0.08$ ) (Table 3).

Non-diabetic medications The use of proton pump inhibitors (PPI) $(39.2 \%$ vs $39.6 \%, P=0.97)$ and aspirin (22.5\% vs $26.5 \%, P=0.29)$ did not differ among cases and controls, respectively (Table 2 ).

Allergies A history of allergies was not different in cases and in controls $(28.9 \%$ vs $25.0 \%, P=0.32)$. Specifically, asthma $(12.1 \%$ vs $8.6 \%, P=0.19)$, eczema (11.1\% vs $7.6 \%, P=0.17$ ) and hay fever (15.6\% vs $14.5 \%$,

Published by Bioscientifica Ltd 
Table 4 Factors associated with TNM stage and tumor grade in PNEN patients.

\begin{tabular}{lcc} 
& & TNM Stage 1-2 N (\%) \\
\cline { 1 - 1 } Late-onset diabetes ( $\geq 1$ year) & & $12(11.8)$ \\
Coffee drinking & & $96(92.3)$ \\
Diabetes & & G1-G2 N $(\%)$ \\
Late-onset diabetes ( $\geq 1$ year) & & $26(15.8)$ \\
Metformin plus insulin use & & $5(3.2)$ \\
Allergic factors & & $18(10.2)$ \\
Asthma & & $17(9.6)$ \\
Eczema & &
\end{tabular}

$P=0.79)$ were not more prevalent in PNEN patients than in controls (Table 2).

\section{Risk factors for the advanced grades and stages of PNEN}

When stratifying cases for the TNM stage at diagnosis and for the tumor grade, diabetes mellitus was statistically more prevalent in patients with G3 tumors (pancreatic neuroendocrine carcinoma; PNEC) than with G1 or G2 tumors (40.9\% vs $15.8 \%, P=0.01$ ). Among cases, non-recent onset diabetes was associated with a more advanced stage at diagnosis (TNM III-IV vs TNM I-II, respectively, 23.3\% vs $11.8 \%, P=0.05)$ and with a G3 vs G1-2 tumor $(40.9 \%$ vs $14.9 \%, P=0.006$, respectively). The use of metformin in combination with insulin was more prevalent in patients with G3 than G1-G2 tumors $(23.5 \%$ vs $3.2 \%, P=0.003$, respectively) (Table 4). Asthma was more prevalent in G3 cases than in $\mathrm{G} 1-2(30.0 \%$ vs $10.2 \% P=0.02)$, and eczema was also more prevalent in G3 cases than in G1-2 but without reaching statistical significance $(25.0 \%$ vs $9.6 \%$, $P=0.08)$. Coffee drinking was more prevalent in localized disease (TNM 1-2) at diagnosis compared with advanced stage (TNM 3-4) $(92.3 \%$ vs $75.9 \%, P=0.003)$.

\section{Discussion}

The present study was designed to recruit cases of PNEN from multiple sites across Europe using a standardized questionnaire in a face-to-face interview setting. To further strengthen our method, we incorporated a preliminary power calculation based on results from a similar previous study. To the best of our knowledge, there have been six published studies to have investigated risk factors for PNEN. Due to the low incidence of such tumors, which would make a longitudinal cohort study highly problematic, it is not unexpected that these were all case-control studies. Three were conducted in the USA, one in Europe and two in China (Hassan et al. 2008a,b Capurso et al. 2009, Zhan et al. 2013, Halfdanarson et al. 2014, Ben et al. 2016). Collective analysis of these studies has been limited by the various data collection methods employed, the selection of investigated exposures (and differing definitions), in addition to the disparate population pools, all of which lead to substantial heterogeneity (Haugvik et al. 2015). One study from China exclusively investigated a cohort of insulinomas, with the exclusion of non-functioning endocrine tumors; the latter represent the majority of PNEN cases, accounting for 60 to $90 \%$ of cases (Falconi et al. 2016). Another study from China investigated a cohort of PNEN cases in which $63 \%$ had functioning tumors, and $84 \%$ had earlystage disease (Ben et al. 2016). The results of this study may have limited applicability to other populations, as the majority of PNENs arising in Western populations are non-functioning and would typically present with more advanced disease (Panzuto et al. 2011).

The two publications from the USA reported different risk factors in the same population, from a retrospective analysis of a large hospital database of NETs (Hassan et al. $2008 a, b)$. None of the reported studies sought to investigate potentially protective factors against the occurrence of PNENs. Two meta-analyses have summarized the results of the previous primary studies and reached similar conclusions: Diabetes mellitus and family history of cancer are risk factors for the occurrence of PNENs, while the role of environmental factors was unclear and warranted further investigation (Haugvik et al. 2015, Leoncini et al. 2016).

Our study affirms an increased risk of PNEN occurrence with diabetes mellitus; however, it is noteworthy that we identify the significance of non-recent onset diabetes as a risk factor. Four studies previously identified an association between PNEN and diabetes (Hassan et al. 2008b, Capurso et al. 2009, Halfdanarson et al. 2014, 
Ben et al. 2016); however, in contrast to the current study, this association was for recent onset diabetes, which can represent an epiphenomenon of the disease as suggested elsewhere for pancreatic adenocarcinoma (Pannala et al. 2008).

Given that beta cells typically express low levels of cytoprotective antioxidant enzymes (Tiedge et al. 1997) and because oxidative stress contributes to both the pathogenesis of diabetes (Rolo \& Palmeira 2006) and can potentiate somatic mutations, it would not be unexpected for long-standing diabetes to have an association with oncogenic transformation of islet cells. Indeed, PNEN proliferation, tumor invasion and disease stage have been found to be associated with expression of mTOR (mechanistic/mammalian target of rapamycin) and its effectors (Capurso et al. 2015), a cytoplasmic kinase that is activated by both glucose and insulin (Blagosklonny 2013).

As the relation between diabetes and carcinogenesis is complex and still not clear, we sought to specifically investigate the timing of onset of diabetes in respect to the clinical presentation of the cancer. We therefore analyzed risk factors present at least 12 months before diagnosis, minimizing the overlap between risk factors and cancerrelated symptoms, which could include cancer-induced endocrine insufficiency. With such premises, our results support the view that long-standing diabetes is a risk factor for PNEN rather than sign of disease. In order to further analyze possible overlaps between diabetes as a risk factors and diabetes as a cancer-related symptom, we performed a further sensitivity analysis, investigating several different intervals of time between the onset of diabetes and the diagnosis of cancer. Non-recent onset diabetes was confirmed to be increasingly consistent with the occurrence of PNEN for intervals superior to 1 year and up to 5 years. For intervals of onset of diabetes superior to 5 years, this association was not anymore statistically significant. This might be interpreted on the base of a lack of power of the study when considering small subgroups or, alternatively, it could be biologically explained by the trophic influence that diabetes plays on cancer. On the other hand, one should also take into account that PNEN displays a slower growing rate compared to PDAC, and therefore it might justify a major latency of occurrence of symptom diabetes.

As the potential role of anti-diabetic drugs (metformin and insulin) in influencing pancreatic carcinogenesis has been reported (De Souza et al. 2016), we also specifically investigated the role of such drugs in our multinational cohort. Although the prevalent use of insulin alone was more frequent among cases than in controls (4.1\% vs $1.6 \%)$, this difference was not significant as the study was underpowered to assess it.

Another noteworthy finding of the present study was the increased proportion of gallstone disease among PNEN cases compared to controls. However, this did not reach statistical significance, and we therefore cannot conclude that this was anything more than a chance observation. The apparent proportional increase among cases, however, may reflect the universal use of abdominal imaging in those diagnosed with PNEN, as compared to occult gallstones in controls. It may be that our study was underpowered to detect a true association between PNEN and biliary calculi, as the latter have been found to be associated with 'malignant neoplasm of the pancreas' (ICD-Oncology C25.0-C25.9) as a single entity, using a large combined US cancer registry with population-based controls (Nogueira et al. 2014).

In the present study, the rate of family history of cancer was not different between cases and controls. This finding was in contrast with previous studies on this topic. However, study design issues and/or selection bias in previous studies may account for this difference. For example, three of these reports (Hassan et al. 2008a,b, Zhan et al. 2013) did not exclude PNEN cases with genetic syndromes (i.e. MEN1 or VHL). Of particular note, 25\% of PNEN cases had a genetic syndrome in the study by Zhan and coworkers, and therefore a higher proportion of family history of any cancer would be expected (Zhan et al. 2013). Halfdanarson and coworkers studied only sporadic cases, but excluded insulinomas and poorly differenced PNECs which may have affected results in this regard (Halfdanarson et al. 2014). In the present study, controls had a significantly higher number of siblings compared with cases, potentially biasing the probability of cancer family history in the control study arm. On the other hand, we found an association between 2nd degree family history of cancer and risk of PNEN, thus suggesting that some kind of hereditary component might exist in these patients.

Environmental factors such as smoking and alcohol, even in high doses, did not increase the risk of developing a PNEN in our study. This result is in keeping with the study by Hassan and coworkers (Hassan et al. $2008 b$ ) but in contrast with others (Capurso 2009, Halfdanarson et al. 2014, Zhan et al. 2013). A recent metaanalysis highlighted that the role of smoking and alcohol might be less relevant in PNENs than in pancreatic 
adenocarcinoma (Haugvik et al. 2015). To explore potential environmental factors that might alter the risk of PNEN occurrence, we investigated for the first time a number of factors associated with a lower incidence of pancreatic adenocarcinoma, such as the use of aspirin and a personal history of allergies. No statistically significant differences were detected, possibly reflecting intrinsic biological differences between endocrine and exocrine neoplasia of the pancreas. Furthermore, as the power of the present study was based on risk factors for which there were previous data, and this was not the case for previously uninvestigated exposures, a type II error may have occurred.

Finally, we investigated the possible prognostic relevance of the investigated factors, analyzing their distribution in PNEN patients according to their stage of disease at diagnosis (TNM stages III or IV compared with I and II) or with their grade assessed by proliferative activity (G1 and G2 compared to G3). Interestingly, the prevalence of non-recent onset diabetes was higher both in cases with metastatic disease (TNM stage III-IV) and advanced grade (G3) at the time of diagnosis (Table 4). Drinking coffee was more frequent in cases with localized disease at diagnosis. The use of metformin in combination with insulin was also associated with a more aggressive phenotype. Therefore, diabetes and use of insulin might also exert a proliferative effect on tumor progression, as reported for other cancer types (Vigneri et al. 2016).

The present study displays several strengths as well as limitations. The strengths of the study are represented by the relatively large sample size keeping in mind the low incidence of this tumor type, the European multicenter setting (6 countries involved), the preliminary power calculation, the investigation for the first time of a large set of factors possibly associated with the risk of PNENs and the conduct of the study by face-to-face interview with a standardized questionnaire. The inclusion criteria were clearly defined: controls were well matched for age and gender with a 1:3 ratio, and all questionnaires were administered by trained medical doctors fluent in the local language, who evaluated exposures present 12 months before diagnosis, to minimize bias due to cancer symptoms. Inherent with a multinational casecontrol design, our study displays some limitations such as potential recall bias and heterogeneity in data from different countries, although the analysis was corrected for centere of enrollment. Furthermore, the analysis might have been underpowered for some of the investigated factors, and additional studies might be important to confirm the lack of significant association.
Another important matter of concern, as for any case-control study, regards the choice of the control population. We opted for a mixed control group that we believed to represent the same population as the case group, as living in the same catchment area of the corresponding cases, to limit possible bias that could have been specific of either hospital controls or visitors. Interestingly, 'late onset diabetes' seemed to be associated with an increased risk of PNEN with both kind of controls used in separate analyses.

In conclusion, the findings of this large multicenter case-control study suggest that non-recent onset diabetes was associated with an increased risk of PNENs occurrence. Our results do not support the view of a strict similarity with factors affecting the risk of pancreatic adenocarcinoma.

\section{Declaration of interest}

The authors declare that there is no conflict of interest that could be perceived as prejudicing the impartiality of this research.

\section{Funding}

Gabriele Capurso was supported by AIRC grant IG 2015, Id 17177.

\section{Author contribution statement}

Gabriele Capurso was the guarantor of the article. All authors approved the final version.

Roberto Valente, Gabriele Capurso, Alastair Hayes. Analyzed the data: Roberto Valente, Gabriele Capurso, Patrick Maisonneuve wrote the paper. All authors contributed to the design, data collection, data interpretation and writing of the manuscript.

\section{Acknowledgements}

This study was conducted through Pancreas 2000, which is a European educational and scientific pancreatology program initiated by the Karolinska Institutet in Stockholm, Sweden, and the European Pancreatic Club. The authors wish to acknowledge the contributions from the following sites: Italy: Dr Livia Archibugi, Dr Maria Rinzivillo. United Kingdom: Dr Lucy Wall, Dr Karen M Hayes, Christos Skouras, Prof. Rowan W Parks, Prof. Mark W J Strachan (Edinburgh); Stuart M Robinson, Colin H Wilson (Newcastle); Tani Fasih, Cho Ee Ng (Gateshead). Norway: Prof. Ivar P Gladhaug, Kjerstin Skrede Mordal. Sweden: Dr Louise Bexander, Dr Isabel Sjöholm and Medical Student Anna Sjögren.

\section{References}

Ben Q, Zhong J, Fei J, Chen H, Yv L, Tan J \& Yuan Y 2016 Risk factors for sporadic pancreatic neuroendocrine tumors: a case-control study. Scientific Reports 6 36073. (doi:10.1038/srep36073)

Blagosklonny MV 2013 TOR-centric view on insulin resistance and diabetic complications: perspective for endocrinologists and gerontologists. Cell Death Discovery 4 e964.

Published by Bioscientifica Ltd. 
Capurso G, Falconi M, Panzuto F, Rinzivillo M, Boninsegna L, Bettini R, Corleto V, Borgia P, Pederzoli P, Scarpa A, et al. 2009 Risk factors for sporadic pancreatic endocrine tumors: a case-control study of prospectively evaluated patients. American Journal of Gastroenterology 104 3034-3041. (doi:10.1038/ajg.2009.466)

Capurso G, Archibugi L \& Delle Fave G 2015 Molecular pathogenesis and targeted therapy of sporadic pancreatic neuroendocrine tumors. Journal of Hepato-Biliary-Pancreatic Sciences 22 594-601. (doi:10.1002/jhbp.210)

De Souza A, Khawaja KI, Masud F \& Saif MW 2016 Metformin and pancreatic cancer: is there a role? Cancer Chemotherapy and Pharmacology 77 235-242. (doi:10.1007/s00280-015-2948-8)

Del Chiaro M, Verbeke C, Salvia R, Kloppel G, Werner J, McKay C, Friess H, Manfredi R, Van Cutsem E, Lohr M, et al. 2013 European experts consensus statement on cystic tumours of the pancreas. Digestive and Liver Disease 45 703-711. (doi:10.1016/j.dld.2013.01.010)

Ellison TA, Wolfgang CL, Shi C, Cameron JL, Murakami P, Mun LJ, Singhi AD, Cornish TC, Olino K, Meriden Z, et al. 2014 A single institution's 26-year experience with nonfunctional pancreatic neuroendocrine tumors: a validation of current staging systems and a new prognostic nomogram. Annals of Surgery 259 204-212. (doi:10.1097/SLA.0b013e31828f3174)

Falconi M, Eriksson B, Kaltsas G, Bartsch DK, Capdevila J, Caplin M, Kos-Kudla B, Kwekkeboom D, Rindi G, Kloppel G, et al. 2016 ENETS consensus guidelines update for the management of patients with functional pancreatic neuroendocrine tumors and non-functional pancreatic neuroendocrine tumors. Neuroendocrinology 103 153-171. (doi:10.1159/000443171)

Fitzgerald TL, Hickner ZJ, Schmitz M \& Kort EJ 2008 Changing incidence of pancreatic neoplasms: a 16-year review of statewide tumor registry. Pancreas 37 134-138. (doi:10.1097/MPA.0b013e318163a329)

Gomez-Rubio P, Zock JP, Rava M, Marquez M, Sharp L, Hidalgo M, Carrato A, Ilzarbe L, Michalski C, Molero X, et al. 2017 Reduced risk of pancreatic cancer associated with asthma and nasal allergies. Gut $66314-322$.

Halfdanarson TR, Bamlet WR, McWilliams RR, Hobday TJ, Burch PA, Rabe KG \& Petersen GM 2014 Risk factors for pancreatic neuroendocrine tumors: a clinic-based case-control study. Pancreas 43 1219-1222. (doi:10.1097/MPA.0000000000000234)

Hassan MM, Phan A, Li D, Dagohoy CG, Leary C \& Yao JC 2008a Family history of cancer and associated risk of developing neuroendocrine tumors: a case-control study. Cancer Epidemiology, Biomarkers and Prevention 17 959-965. (doi:10.1158/1055-9965.EPI-07-0750)

Hassan MM, Phan A, Li D, Dagohoy CG, Leary C \& Yao JC $2008 b$ Risk factors associated with neuroendocrine tumors: a U.S.-based case-control study. International Journal of Cancer 123 867-873. (doi:10.1002/ijc.23529)

Haugvik SP, Hedenstrom P, Korsaeth E, Valente R, Hayes A, Siuka D, Maisonneuve P, Gladhaug IP, Lindkvist B \& Capurso G 2015 Diabetes, smoking, alcohol use, and family history of cancer as risk factors for pancreatic neuroendocrine tumors: a systematic review and meta-analysis. Neuroendocrinology 101 133-142. (doi:10.1159/000375164)

Jensen RT, Cadiot G, Brandi ML, de Herder WW, Kaltsas G, Komminoth P, Scoazec JY, Salazar R, Sauvanet A \& Kianmanesh R 2012 ENETS consensus guidelines for the management of patients with digestive neuroendocrine neoplasms: functional pancreatic endocrine tumor syndromes. Neuroendocrinology 95 98-119. (doi:10.1159/000335591)

Leoncini E, Carioli G, La Vecchia C, Boccia S \& Rindi G 2016 Risk factors for neuroendocrine neoplasms: a systematic review and metaanalysis. Annals of Oncology 27 68-81. (doi:10.1093/annonc/mdv505)

Lepage C, Bouvier AM, Phelip JM, Hatem C, Vernet C \& Faivre J 2004 Incidence and management of malignant digestive endocrine tumours in a well defined French population. Gut 53 549-553. (doi:10.1136/gut.2003.026401)

Nogueira L, Freedman ND, Engels EA, Warren JL, Castro F \& Koshiol J 2014 Gallstones, cholecystectomy, and risk of digestive system cancers. American Journal of Epidemiology 179 731-739. (doi:10.1093/aje/ kwt322)

Pannala R, Leirness JB, Bamlet WR, Basu A, Petersen GM \& Chari ST 2008 Prevalence and clinical profile of pancreatic cancer-associated diabetes mellitus. Gastroenterology 134 981-987. (doi:10.1053/j. gastro.2008.01.039)

Panzuto F, Boninsegna L, Fazio N, Campana D, Pia Brizzi M, Capurso G, Scarpa A, De Braud F, Dogliotti L, Tomassetti P, et al. 2011 Metastatic and locally advanced pancreatic endocrine carcinomas: analysis of factors associated with disease progression. Journal of Clinical Oncology 29 2372-2377. (doi:10.1200/JCO.2010.33.0688)

Rindi GAR, Bosman F, Capella C, Klimstra D, Klöppel G, Komminoth P \& Solcia E 2010 Nomenclature and classification of neuroendocrine neoplasms of the digestive system. In WHO Classification of Tumours of the Digestive System.

Rolo AP \& Palmeira CM 2006 Diabetes and mitochondrial function: role of hyperglycemia and oxidative stress. Toxicology and Applied Pharmacology 212 167-178. (doi:10.1016/j.taap.2006.01.003)

Solcia E, Klöppel G, Sobin LH \& World Health Organization 2002 Histological typing of endocrine tumors. In Collaboration With 9 Pathologists From 4 Countries, 2nd edn, pp 1-5. Berlin, Heidelberg, New York: Sprinter Verlag.

Streicher SA, Yu H, Lu L, Kidd MS \& Risch HA 2014 Case-control study of aspirin use and risk of pancreatic cancer. Cancer Epidemiology, Biomarkers and Prevention 23 1254-1263. (doi:10.1158/1055-9965. EPI-13-1284)

Tiedge M, Lortz S, Drinkgern J \& Lenzen S 1997 Relation between antioxidant enzyme gene expression and antioxidative defense status of insulin-producing cells. Diabetes 46 1733-1742. (doi:10.2337/diab.46.11.1733)

Vigneri R, Goldfine ID \& Frittitta L 2016 Insulin, insulin receptors, and cancer. Journal of Endocrinological Investigation 39 1365-1376. (doi:10.1007/s40618-016-0508-7)

Yao JC, Hassan M, Phan A, Dagohoy C, Leary C, Mares JE, Abdalla EK, Fleming JB, Vauthey JN, Rashid A, et al. 2008 One hundred years after 'carcinoid': epidemiology of and prognostic factors for neuroendocrine tumors in 35,825 cases in the United States. Journal of Clinical Oncology 26 3063-3072. (doi:10.1200/JCO.2007.15.4377)

Zhan HX, Cong L, Zhao YP, Zhang TP \& Chen G 2013 Risk factors for the occurrence of insulinoma: a case-control study. Hepatobiliary and Pancreatic Diseases International 12 324-328. (doi:10.1016/S1499. 3872(13)60051-X)

Received in final form 12 May 2017

Accepted 30 May 2017

Accepted Preprint published online 31 May 2017 http://erc.endocrinology-journals.org DOI: $10.1530 /$ ERC-17-0040
C 2017 Society for Endocrinology Printed in Great Britain
Published by Bioscientifica Ltd. 